\title{
La remuneración según el desempeño no asegura mejoras en el ámbito sanitario
}

Pay for performance does not guarantee improvements in the health field

\section{Objetivos}

Evaluar la efectividad de la remuneración por desempeño sobre el trabajo de los médicos.

\section{Fuentes de datos}

MEDLINE, EMBASE, Cochrane Library, OpenSIGLE, Canadian Evaluation Society Unpublished Literature Bank, New York Academy of Medicine Library Grey Literature Collection, y listas de referencias hasta junio 2012.

\section{Selección de estudios}

Ensayos controlados y aleatorizados, series de tiempo interrumpidas, estudios antes-después controlados y no controlados, y grupos de cohortes comparativas.

\section{Extracción de datos}

Los datos fueron extraídos por dos revisores independientes.

\section{Resultados principales}

La tabla 1 resume los efectos del pago por desempeño sobre la tasa de implementación de cuidados preventivos y/o maniobras de rastreo y la tabla 2, sus efectos sobre la calidad del cuidado en pacientes con enfermedades crónicas (ambas tablas fueron resumidas, otorgándole mayor importancia a los estudios más robustos).

Tabla 1: Efectos del pago por desempeño sobre la tasa de implementación de cuidados preventivos y/o maniobras de rastreo.

\begin{tabular}{|c|c|c|c|c|c|}
\hline \multirow{2}{*}{$\begin{array}{l}\text { Autor y año de } \\
\text { publicación }\end{array}$} & \multirow{2}{*}{$\begin{array}{l}\text { Diseño del } \\
\text { estudio }\end{array}$} & \multirow{2}{*}{$\begin{array}{l}\text { Indicadores comparados } \\
\text { (tasas de) }\end{array}$} & \multicolumn{3}{|c|}{ Resultados por grupo } \\
\hline & & & Control & Intervención & Significancia estadistica \\
\hline $\begin{array}{l}\text { Fairbrother y col., } 2001 \\
\text { Kouides y col., } 1998 \\
\text { Grady y col., } 1997\end{array}$ & $\begin{array}{c}\text { Ensayo clínico controlado } \\
\text { aleatorizado }\end{array}$ & $\begin{array}{l}\text { Vacunación en niños } \\
\text { Inmunización contra la influenza } \\
\text { Indicación de mamografía } \\
\text { Rastreo con mamografía }\end{array}$ & \begin{tabular}{|l}
$2,5 \%$ \\
$2,5 \%$ \\
$25,0 \%$ \\
$30,2 \%$
\end{tabular} & $\begin{array}{c}5,9 \% \\
10,3 \% \\
26,0 \% \\
28,2 \%\end{array}$ & $\begin{array}{l}p<0,05 \\
p=0,03 \\
p=0,46 \\
P=0,14\end{array}$ \\
\hline Gavagan y col., 2010 & $\begin{array}{c}\text { Ensayo controlado no } \\
\text { aleatorizado }\end{array}$ & $\begin{array}{l}\text { Rastreo con Papanicolaou } \\
\text { Rastreo con mamografía }\end{array}$ & $\begin{array}{c}\text { Tendencias: } 0,004 \\
0,0015\end{array}$ & $\begin{array}{c}\text { Tendencias: } 0,005 \\
0,003\end{array}$ & $\begin{array}{l}p<0.053 \\
p<0,076\end{array}$ \\
\hline $\begin{array}{l}\text { Rosenthal y col., } \\
2005\end{array}$ & $\begin{array}{l}\text { Controlado antes (A)- } \\
\text { después (D) }\end{array}$ & $\begin{array}{c}\text { Rastreo de cáncer cervical } \\
\text { Rastreo con mamografía } \\
\text { Alcance de la meta de hemoglobina } \\
\text { glicosilada }^{c}\end{array}$ & $\begin{array}{c}\text { A: } 39 \% ; D: 55 \% \\
\text { A: } 66,1 \% ; D: 72,4 \% \\
\text { A: } 62 \% ; C: 80 \%\end{array}$ & $\begin{array}{l}\text { A: } 44 \% ; D: 57 \% \\
\text { A: } 68,0 \% ; D: 72,6 \% \\
\text { A: } 64, \% ; D: 82,1 \%\end{array}$ & $\begin{array}{l}p=0,02 \\
p=0,13 \\
p=0,50\end{array}$ \\
\hline Fagan y col., 2010 & $\begin{array}{l}\text { Estudio controlado } \\
\text { antes-después }\end{array}$ & $\begin{array}{c}\text { Rastreo de retinopatía } \\
\text { Rastreo de nefropatía } \\
\text { Inmunización contra influenza } \\
\text { Alcance de la meta de hemoglobina } \\
\text { glicosiladac } \\
\text { Alcance de la meta de colesterol LDL }\end{array}$ & No reportado & & $\begin{array}{l}\text { OR: } 0,98(0,61 \text { a } 1,58) \\
\text { OR: } 0,95(0,62 \text { a } 1,46) \\
\text { OR: } 1,79(1,37 \text { a } 2,35) \\
\text { OR: } 0,44(0,30 \text { a } 0,65) \\
\text { OR: } 0,62(0,44 \text { a } 0,86)\end{array}$ \\
\hline
\end{tabular}

A: antes. D: después. C: en personas con diabetes.

Tabla 2: Efectos del pago por desempeño sobre pacientes con condiciones crónicas.

\begin{tabular}{|c|c|c|c|c|c|}
\hline \multirow{2}{*}{$\begin{array}{l}\text { Autor y año de } \\
\text { publicación }\end{array}$} & \multirow{2}{*}{$\begin{array}{l}\text { Diseño del } \\
\text { estudio }\end{array}$} & \multirow{2}{*}{$\begin{array}{l}\text { Indicadores comparados } \\
\text { (tasas de) }\end{array}$} & \multicolumn{3}{|c|}{ Resultados por grupo } \\
\hline & & & Control & Intervención & Significancia estadística \\
\hline Twardella y col. 2007 & Estudio aleatorizado y controlado & $\begin{array}{l}\text { Proporción de pacientes que } \\
\text { reportaron no estar fumando }\end{array}$ & $2,7 \%$ & $3,5 \%$ & $p=0,75$ \\
\hline \multirow[t]{2}{*}{ Campbell y col., 2009} & \multirow{2}{*}{$\begin{array}{l}\text { Serie de tiempo interrumpida } \\
\text { comparando la mejoría } \\
\text { "atribuible" a la intervención de } \\
\text { pago por desempeño con la } \\
\text { mejoría observada en períodos } \\
\text { históricos durante los cuales no } \\
\text { se habían implementado } \\
\text { intervenciones semejantes. }\end{array}$} & $\begin{array}{l}\text { Cuidados apropiados en pacientes } \\
\text { con enfermedad coronaria }\end{array}$ & $\begin{array}{c}\text { De } 58,6 \% \text { en } 1998 \\
\text { a } 76,2 \% \text { en } 2003 \\
p=0,6\end{array}$ & $\begin{array}{c}\text { De } 85,0 \% \text { en } 2005 \\
\text { a } 84,8 \% \text { en } 2007 \\
p=0,02\end{array}$ & \\
\hline & & $\begin{array}{l}\text { Cuidados apropiados en pacientes } \\
\text { con asma } \\
\text { Cuidados apropiados en pacientes } \\
\text { con enfermedad diabetes }\end{array}$ & $\begin{array}{c}\text { De } 60,2 \% \text { en } \\
1998 \text { a } 70,3 \% \text { en } \\
2003 \\
p=0,001 \\
\text { De } 61,6 \% \text { en } \\
1998 \text { a } 70,4 \% \text { en } \\
2003 \\
p<0.001\end{array}$ & $\begin{array}{c}\text { De } 84,3 \% \text { en } \\
2005 \text { a } 85 \% \text { en } \\
2007 \\
p=0,16 \\
\text { De } 81,4 \% \text { en } \\
2005 \text { a } 83,7 \% \text { en } \\
2007 \\
p=0,91\end{array}$ & \\
\hline
\end{tabular}




\begin{tabular}{|c|c|c|c|c|c|}
\hline \multirow[t]{4}{*}{ Serumaga y col., 2011} & \multirow{4}{*}{$\begin{array}{c}\text { Serie de tiempo interrumpida en } \\
\text { pacientes con hipertensión } \\
\text { arterial }\end{array}$} & $\begin{array}{c}\text { Pacientes que alcanzaron valores } \\
\text { menores o iguales a } 150 / 90 \mathrm{mmHg}\end{array}$ & $70 \%$ & $67,3 \%$ & $\begin{array}{l}-0.01 \%(-0.06 \text { a } 0.03) \text { de } \\
\text { cambio en la tendencia }\end{array}$ \\
\hline & & Controles de la tensión arterial & $47,7 \%$ & $53,2 \%$ & $\begin{array}{l}-0.01 \%(-0.24 \text { a } 0,21) \text { de } \\
\text { cambio en la tendencia }\end{array}$ \\
\hline & & $\begin{array}{l}\text { Pacientes que iniciaron tratamiento } \\
\text { con nuevo fármaco }\end{array}$ & $0,05 \%$ por mes & $0,05 \%$ por mes & $\begin{array}{l}0,02 \%(-0.23 \text { a } 0,19) \text { de } \\
\text { cambio en la tendencia }\end{array}$ \\
\hline & & $\begin{array}{l}\text { Ocurrencia de resultados } \\
\text { relacionados (infarto de miocardio, } \\
\text { ACV, insuficiencia cardiaca, } \\
\text { insuficiencia renal, muerte). }\end{array}$ & No reportada & No reportada & $\begin{array}{l}0,05 \%(-0,02 \text { a } 0,07) \text { de } \\
\text { cambio en la tendencia }\end{array}$ \\
\hline $\begin{array}{l}\text { Vamos y col., } \\
2011\end{array}$ & $\begin{array}{l}\text { Serie de tiempo interrumpida en } \\
\text { pacientes con diabetes. } \\
\text { Se valoró la tasa de mejora } \\
\text { anual de los objetivos a alcanzar. }\end{array}$ & $\begin{array}{c}\text { Presión sanguínea } \\
\text { Colesterol total menor a } 193 \mathrm{mg} / \mathrm{dL} \\
\text { Hemoglobina glicosilada menor } 0 \\
\text { igual a } 7 \%\end{array}$ & $\begin{array}{c}2,2 \%(1.9 \text { a } 2.6) \\
4,9 \%(4,3 \text { a } 5,3) \\
2(1,3 \text { a } 2,)\end{array}$ & $\begin{array}{c}3,8 \%(2,7 \text { a } 4.9) \\
7,4 \%(6 \text { a } 8,8) \\
-0,2 \%(-2 \text { a } 1,6)\end{array}$ & $\begin{array}{c}\text { El pago por desempeño se } \\
\text { asoció a una mejoría } \\
\text { estadísticamente significativa } \\
\text { de los dos primeros indicadores } \\
\text { y a un empeoramiento del } \\
\text { tercero. }\end{array}$ \\
\hline $\begin{array}{l}\text { Alshamsan y col., } \\
2012\end{array}$ & $\begin{array}{l}\text { Serie de tiempo en pacientes } \\
\text { con diabetes }\end{array}$ & $\begin{array}{l}\text { Hemoglobina glicosilada } \\
\text { Colesterol total } \\
\text { Presión Sistólica }\end{array}$ & No reportada & No reportada & $\begin{array}{c}\text { Cambios en la tendencia: } \\
-0,19 \% \\
(0,15 \text { a } 0,22) \\
-0,03 \mathrm{mmol} / \mathrm{L} \\
(0,01 \text { a } 0,05) \\
-1,04 \mathrm{mmHg} \\
(-1,42 \mathrm{a}-0,64)\end{array}$ \\
\hline
\end{tabular}

ACV: accidente cerebrovascular.

\section{Conclusiones}

Si bien el pago atado al desempeño ha demostrado ser útil en el ámbito de los negocios, todavía no contamos con evidencia suficiente como para recomendar aplicarlo en sistemas de salud.
Fuente de financiamiento: Houle S recibió apoyo para sus estudios de doctorado de las siguientes instituciones: Interdisciplinary Chronic Disease Collaboration, Hypertension Canada, Canadian Institutes for Health Research. McAlister F cobró honorarios de: Alberta Innovates Health Solutions y Capital Health Chair in Cardiovascular Outcomes Research. Posibles conflictos de interés: No referidos.

\section{Comentario}

El incentivo financiero es una metodología discutida para mejorar la calidad del trabajo médico, sobre todo porque no ha terminado de demostrar fehacientemente su efectividad en la práctica ${ }^{1}$

En Argentina solo hemos podido identificar dos experiencias de este método de remuneración.

Por un lado, en 2007 Giménez $L$ y col. ${ }^{2}$ comunicaron que la implementación de incentivos financieros en una empresa de medicina prepaga se asoció a mejoras en la aceptabilidad de los usuarios medidas a través del cuestionario CAHPS (Consumer Assessment of Healthcare Providers and Systems). Por otro lado, en el Servicio de Medicina Familiar y Comunitaria del Hospital Italiano de Buenos Aires funciona desde $2005^{3}$ un sistema de incentivos financieros atado al puntaje obtenido a través de una mezcla de resultados determinados por consenso entre los médicos del servicio que incluye indicadores de efectividad clínica, de compromiso institucional y de realización de procedimientos vinculados a dicha especialidad. Vale destacar que en 2007 se observó una mejoría estadísticamente significativa en la mayoría de los indicadores de efectivi- dad clínica: aumento de la tasa de rastreo de cáncer colorrectal y de mama, en los niveles de hemoglobina glicosilada en la población con diabetes y de colesterol LDL en la población con enfermedad coronaria o con alto riesgo cardiovascular y en el control de la tensión arterial.

\section{Conclusiones del comentador}

Si bien los resultados iniciales obtenidos de su aplicación en Argentina son alentadores, consideramos que el entusiasmo por el pago por desempeño como un motor de mejoras de calidad en salud es desproporcionado para la actual evidencia disponible. Necesitamos más estudios robustos antes de recomendarlo en forma segura como un potente instrumento para modificar conductas y mejorar resultados. Alentamos además a las organizaciones que actualmente los están utilizando a publicar sus hallazgos con el objetivo de contribuir a ampliar la evidencia científica sobre el tema.

Fernando Ramón Vázquez Peña [ Servicio de Medicina Familiar y Comunitaria, Hospital Italiano de Buenos Aires. fermandoramon.vazquez@hospitalitaliano.org.ar ]

Vázquez Peña F. La remuneración según el desempeño no asegura mejoras en el ámbito sanitario. Evid Act Pract Ambul. Ene-Mar 2013. 16(1). 1213. Comentado de: Houle $S$ y col. Does Performance-Based Remuneration for Individual Health Care Practitioners Affect Patient Care? Ann Intern Med 2012;157:889-899.

\section{Referencias}

1. Rubinstein A., Mejoramiento de calidad e incentivos financieros en atención primaria de la salud. Seminario III, CEDES (Centro de estudios de estado y sociedad), 2005. Disponible en: http://www.cedes.org.ar/Publicaciones/SSPP/2005/SSPP200503.pdf (ultimo acceso: 19/03/13).

2. Giménez L y col. Experiencia de nueve años de trabajo en una aseguradora de Medicina Prepaga de Argentina (OMINT: Organización Médica Internacional). Presentación en la Conferencia Internacional Nro 24 de ISQua (Intemational Society for Quality in Healthcare). Boston (2007).

3. Rubinstein A y col. A multimodal strategy based on pay-per-performance to improve quality of care of family practitioners in Argentina. J Ambulatory Care Manage 2009, Vol. 32, No. 2, pp. 103-114. Disponible en URL: http:/www.mendeley.com/catalog/multimodal-strategy-based-pay-per-performance-improve-quality-care-family-practitioners-argentina/ (ultimo acceso: 19/03/13) 